\section{LINER OPTIMIZATION STUDIES USING THE DUCTED FAN NOISE PREDICTION CODE TBIEM3D}

\author{
M.H. Dunn* \\ Department of Mathematics and Statistics \\ Old Dominion University, Norfolk, Virginia \\ F. Farassat ${ }^{\dagger}$ \\ NASA Langley Research Center, Hampton, Virginia \\ Presented at the 4th AIAA/CEAS Aeroacoustics \\ Conference \\ June 2-4, 1998, Toulouse, France
}

\section{Abstract}

In this paper we demonstrate the usefulness of the ducted fan noise prediction code TBIEM3D as a liner optimization design tool. Boundary conditions on the interior duct wall allow for hard walls or a locally reacting liner with axially segmented, circumferentially uniform impedance. Two liner optimization studies are considered in which farfield noise attenuation due to the presence of a liner is maximized by adjusting the liner impedance. In the first example, the dependence of optimal liner impedance on frequency and liner length is examined. Results show that both the optimal impedance and attenuation levels are significantly influenced by liner length and frequency. In the second example, TBIEM3D is used to compare radiated sound pressure levels between optimal and non-optimal liner cases at conditions designed to simulate take-off. It is shown that significant noise reduction is achieved for most of the sound field by selecting the optimal or near optimal liner impedance. Our results also indicate that there is a relatively large region of the impedance plane over which optimal or near optimal liner behavior is attainable. This is an important conclusion for the designer since there are variations in liner characteristics due to manufacturing imprecisions.

\footnotetext{
Associate Professor, AIAA Member

${ }^{\dagger}$ Senior Research Scientist, AIAA Associate Fellow

"This paper is declared a work of the United States government and is not subject to copyright protection in the United States.
}

\section{List of Symbols}

Dimensional quantities are denoted by a tilde ( )

$\tilde{c} \quad$ Ambient speed of sound

$k \quad=\left\{\begin{array}{ll}\frac{m \tilde{r_{D}} \tilde{\Omega}}{\tilde{c}} & m \neq 0 \\ \frac{\tilde{r}_{D} \tilde{\Omega}}{\tilde{c}} & m=0\end{array}\right.$ Nondimensional

characteristic wavenumber

$\tilde{L} \quad$ Liner length

$L_{D} \quad$ Ratio of duct length to radius

$m \quad=0, \pm 1, \pm 2, \ldots$ Circumferential mode number

$M \quad$ Inflow Mach number

$M_{\text {TIP }}=\frac{\tilde{r}_{D} \tilde{\Omega}}{\tilde{c}}$ Tip Mach number of fan

$N_{B} \quad$ Number of spinning point dipoles

$\tilde{r}_{D} \quad$ Duct radius

$P_{m} \quad m-t h$ modal coefficient of acoustic pressure

$\tilde{R}_{O B S} \quad$ Observer radius

$\tilde{\rho}_{0} \quad$ Ambient density

$\tilde{\Omega} \quad$ Temporal frequency (radians/second)

\section{Introduction}

One of the most important noise control methods for ducted fans is the use of liners in the bypass duct wall. The selection of liners for optimum noise reduction is usually based on simple models of sound propagation in an infinite duct with or without flow ${ }^{1-4}$. Improvements in these models are needed, particularly for a lined finite duct in forward motion. It appears that advanced passive liners have the potential of further reducing the radiated noise of ducted fans as compared to current engines. New liner designs are under development at present with attenuation over a range of frequency ${ }^{5}$. The required liner characteristics must be established by quantifying the liner attenuation 
for a specitic trequency and duct flow condition. fifficient optimization of the noise attenuation of these liners in realistic engine conditions requires new tools based on complex models yet capable of running on desktop computers.

In this paper, we present some results on liner optimization using a finite duct noise propagation and radiation code. The code is called TBIEM3D (Thinduct Boundary Integral Equation Method, 3 Dimensional) ${ }^{6}$. For a fixed circumferential mode number, the optimization is based on minimizing the integral of the square of the acoustic pressure over an arc of observation points in the radiated farfield. Two examples are given to illustrate the capability of this new ducted fan noise prediction tool.

\section{The Ducted Fan Noise Prediction Code TBIEM3D}

The ducted fan noise prediction code TBIEM3D predicts the noise from an infinitely thin finite length, circular duct with a rigid or lined interior. Uniform axial inflow is allowed. The theory on which the code is based was presented by Dunn, Tweed and Farassat ${ }^{7}$. The sources of sound inside the duct are of point monopole or dipole type. To represent many blades, multiple sources located at equal angles circumferentially, are also allowed. These sources may be rotating or fixed with respect to the duct. The code also treats axially segmented, locally reacting liners in its interior. Any portion of the acoustic field can be computed without calculating the entire field.

Mathematically, the problem of noise propagation in a finite duct and radiation from the inlet and the exhaust is formulated as follows. The governing equation is the linearized convected wave equation for the $m$-th circumferential mode of acoustic pressure. For a given frequency and suitable choice of dependent variable, this equation becomes the Helmholtz equation in the frame fixed to the duct. The acoustic pressure is first split into known incident and the unknown scattered pressures. The solution of the boundary value problem for the scattered pressure is written as integrals of single and double layer potentials over the duct surface. This solution satisfies the Helmholtz equation and the far field radiation (Sommerfeld) condition. Imposition of the boundary conditions on the exterior and the interior of the duct walls results in two coupled hypersingular linear integral equations. To obtain a unique solution, other conditions at the inlet and the exhaust edges of the duct must be satisfied.

The coupled singular integral equations are solved for the single and the double layer source strengths using the collocation method. Once these source strengths are found, the scattered acoustic pressure is calculated everywhere by an integral representation and is then added to the incident acoustic pressure to obtain the total acoustic pressure.

TBIEM3D is designed to execute quickly on a personal computer. As the examples below illustrate, many of the questions of ducted fan engine noise radiation and noise reduction by liners can be answered by this code. Input to the code is simple which facilitates parametric or optimization studies.

\section{Liner Optimization Problem}

We present two applications of TBIEM3D to liner optimization. In the first application, we consider a long duct whose interior is lined with a liner of fixed length. The optimum liner impedance is then found by minimizing the integral of the square of the acoustic pressure along an arc of observers in the acoustic far field by a search technique in the impedance plane. Several values of source frequency are considered. Next we repeat similar calculations at a fixed source frequency for a duct with various lengths of liner. A stationary duct is considered here with a single monopole source inside the duct. This example is based on a NASA publication of Lester and Posey ${ }^{2}$. Our study obtains the results of these authors qualitatively.

In the second application, a short duct with a liner of fixed length on the interior wall is assumed. The duct is in motion at Mach number 0.4. The incident acoustic pressure inside the duct is generated by rotating dipole sources. Again the liner is optimized in the impedance plane by a method similar to that of the first example. The radiation field of the optimized liner is then compared with those of a duct with rigid interior wall as well as ducts with nonoptimum liner characteristics.

\section{Monopole Source Inside a Stationary Lined Duct}

Figure 1 shows the duct configuration for this example. The duct is seven meters long with a monopole source on the axis at five meters from the inlet disc. The long duct length in this example is for reducing the influence of the exhaust noise radiation on the acoustic power in the region ahead of the inlet. The duct radius is one meter and the interior of the duct is lined for a length of two meters near the inlet with the liner leading edge one meter from the inlet. The duct is assumed stationary.

Define the parameter I by the formula

$$
I=I 0 \log _{10}\left[\frac{I}{\theta_{1}-\theta_{0}} \int_{\theta_{0}}^{\theta_{L}}\left|P_{m}^{2}\right| d \theta\right] \text {. }
$$


The integration in equation (1) is over the are of observers as indicated in figure 1. The expression in the square brackets in equation (1) is the square of the $L_{2}$ norm of the acoustic pressure on the observer arc. Thus, $I$ gives the decibel level of the mean square pressure on the arc.

We next define attenuation for a particular impedance value of a lined duct by the relation

$$
\text { Attenuation }=I_{\text {RIGID }}-I_{\text {LNED }} \text {, }
$$

where the subscripts stand for a duct with rigid and lined walls. It is obvious that the $I_{\text {LNED }}$ is minimized if the attenuation is maximized in the optimization process.

The liner optimization is performed by a quasiNewton search technique in the impedance plane. In figure 2, for each value of $k$, we have taken a grid in the impedance plane and then calculated the attenuation at each grid point based on equations (1) and (2). We have taken 75 points on the arc of the circle in figure 1 for evaluating the integral in equation (1).

In figure 2, contours of attenuation are plotted for $k=2.0,4.0,6.0$, and 8.0. For each $k$, there is a region of the impedance plane for which the attenuation is maximum, i.e., the liner is optimum for the particular wave number. For $k=2.0$ and 8.0 , an optimum attenuation of more than $14 \mathrm{~dB}$ can be achieved while the optimum attenuation for $k=4.0$ and 6.0 are about 7 and $9 d B$, respectively. It is seen that the optimum liner impedance is a function of frequency. It is also interesting to note that there is a relatively large region of the impedance plane over which optimal or near optimal liner behavior is attainable. This is good news for liner designers since there are variations in liner characteristics due to manufacturing imprecisions.

Figure 3 shows the attenuation plots for a fixed value of the nondimensional wave number $k=6.0$ for four different liner lengths. In these calculations, the location of the trailing edge of the liner is kept fixed for all liner lengths. It is seen from this figure that the maximum attenuation decreases as the liner length is reduced. This is to be expected on intuitive grounds. The somewhat surprising result is that the optimum liner impedance changes as we reduce the liner length. The change involves essentially a decrease in the value of the reactance towards negative values.

\section{Rotating Dipole Sources Inside a Lined Duct at Forward Flight}

Figure 4 shows the duct and liner configuration for this example. Note that the length to diameter ratio $L_{D}=0.5$ here and the observer arc where we integrate the acoustic pressure includes both inlet and exhaust radiation. A liner of fixed length of 0.4 meters is used on the interior wall as shown in tigure 4 . Incident sound is generated by $N_{B}=20$ spinning dipoles. The dipole axis is in the axial direction. We are interested in liner optimization at the fundamental harmonic $m=$ 20. The duct is in uniform forward flight with $M=$ 0.4. These conditions were chosen to simulate loading noise of an actual fan.

We use the search technique for finding the optimum liner impedance described in the previous section using equations (1) and (2). Figure 5 shows the plot of the attenuation contours in the impedance plane. It is interesting to note that it is possible to get more than $18 \mathrm{~dB}$ of attenuation with the optimum liner impedance. We again note that the region of optimum or near optimum liner attenuation is large in the impedance plane.

Next we look at the effect of liner impedance on the radiation fields of the inlet and the exhaust. We take three values of the impedance as indicated in figure 5. Note that two of the values of the impedance do not lie in the region of the maximum attenuation. In figure 6 we plot sound pressure levels of the radiated noise from the inlet and the exhaust. We have also plotted the radiation field of a geometrically similar duct with rigid wall at the same conditions for comparison. First we note that the ducts with liner radiate less noise from the inlet and the exhaust than the rigid duct. In the inlet region, the duct with the optimum liner has the lowest radiated noise level among the four ducts. The attenuation compared to the rigid duct is impressive. In the exhaust region, surprisingly, the duct with the nonoptimum liner impedance gives the lowest noise radiation. Note that only the region near the inlet is lined. Nevertheless, it is clear that liner optimization depends on many factors such as the length and position of the liner and the region of minimization of the acoustic pressure level.

\section{Concluding Remarks}

In this paper, we have demonstrated the versatility of TBIEM 3D as a liner optimization tool by conducting noise attenuation studies for two cases in which a variety of operating conditions were considered.

The first case was chosen for comparison with the earlier research of Lester and Posey. Calculations presented here agree qualitatively with the results of reference 2 . We show that there is a relatively large region of the impedance plane over which optimal or near optimal liner behavior is attainable Also it is 
demonstrated that the optimal liner impedance is a function of liner length.

In the second example, operating parameters were chosen (1) simulate take-off conditions of a modern turbotan engine. By examining the radiated pressure patterns for several different liner impedances we show that signiticant reduction in farfield SPL is attainable by selecting the optimal or near optimal liner. Furthermore, the exhaust radiation pattern can be seriously affected by the presence of a liner in the inlet duct region.

The definition of noise attenuation used here is somewhat arbitrary and was chosen for its smoothing properties. Other choices (maximum reduction in sound pressure level, for example) that include the peak noise regions in their definitions should yield similar results.

Calculations presented here required several minutes execution time on a personal computer with a Pentium 133 processor and 32 megabytes of RAM. Consequently, large scale parametric studies using TBIEM $3 D$ are feasible. For example, optimizing the effects of liner location or segmented liners on radiated noise levels over a range of frequency can be easily handled.

\section{References}

1. R.E. Motsinger and R. E. Kraft: Design and Performance of Duct Acoustic Treatment, in Aeroacoustics of Flight Vehicles- Theory and Practice, Vol. 2, H. H. Hubbard, NASA RP-1258, 1991

2. Harold C. Lester and Joe W. Posey: Optimal OneSection and Two-Section Circular SoundAbsorbing Duct Liners for Plane-Wave and Monopole Sources without Flow, NASA TN D8348, December 1976

3. Edward J. Rice: Attenuation of Sound in Ducts with Acoustic Treatment - A generalized Approximate Equation, NASA TM X-71830, 1975.

4. Edward J. Rice: Acoustic Liner Optimum Impedance for Spinning Modes with Cut-Off Ratio as the Design Parameter, NASA TM X-73411, 1976.

5. Tony L. Parrott and Michael G. Jones: ParallelElement Liner Impedances for Improved Absorption of Broadband Sound in the Duct, Noise Control Engineering Journal, Vol. 43(6), 1995, p 183-195

6. M.H. Dunn: TBIEM3D - A Computer Program for Predicting Ducted Fan Engine Noise, Version 1.1. NASA/CR-97-206232, September 1997.
7. M.H. Dunn, J. Tweed, and F. Farassat: The Prediction of Ducted Fan Engine Noise Via a Boundary Integral Equation Method. AIAA Paper 96-1770, April, 1996. 


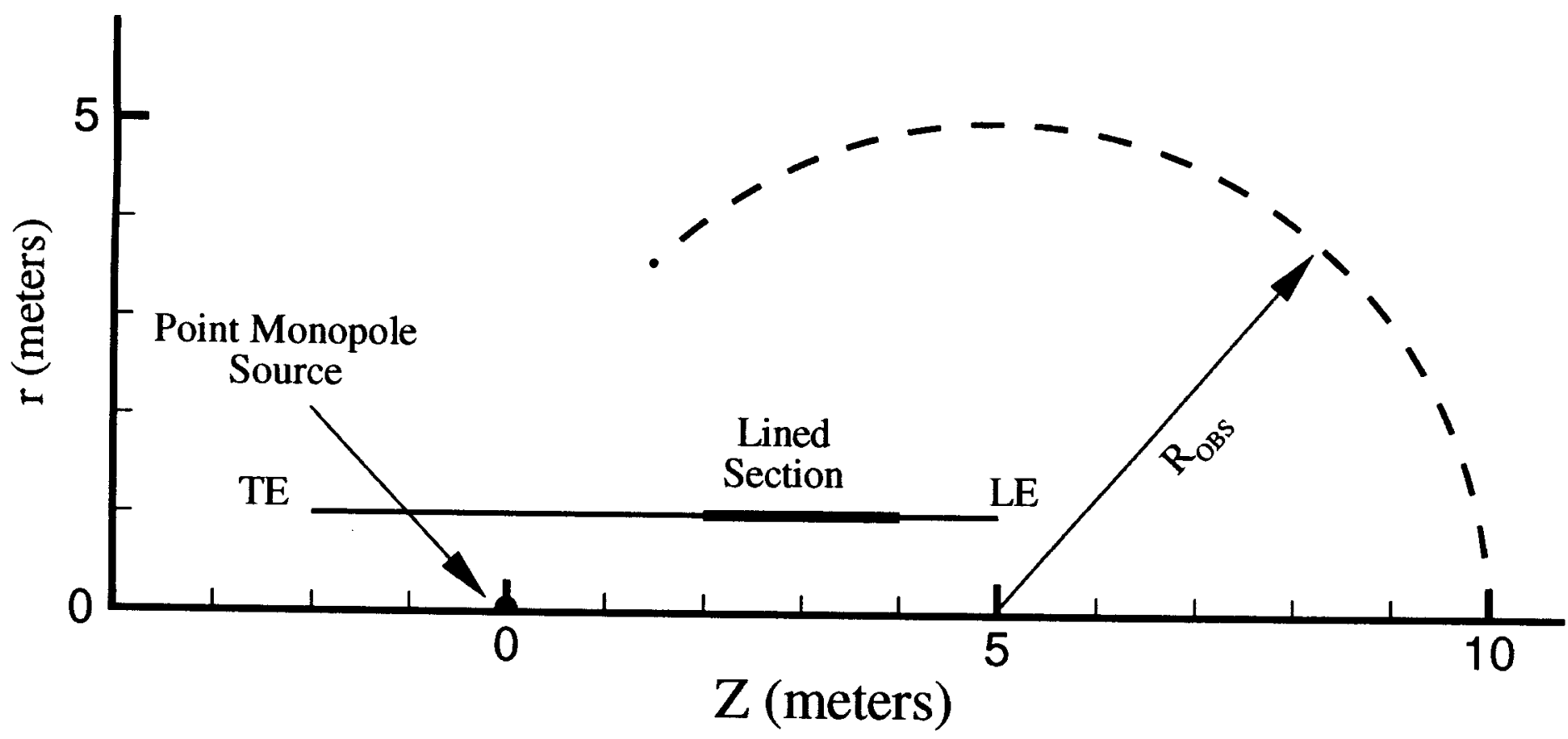

Figure 1: Configuration for Liner Optimization Studies with Point Monopole Source on Duct Axis

$$
\mathrm{m}=0 \quad \mathrm{M}=0.0 \quad \mathrm{~L}_{\mathrm{D}}=3.5 \quad \mathrm{R}_{\mathrm{OBS}}=5.0 \mathrm{~m}
$$



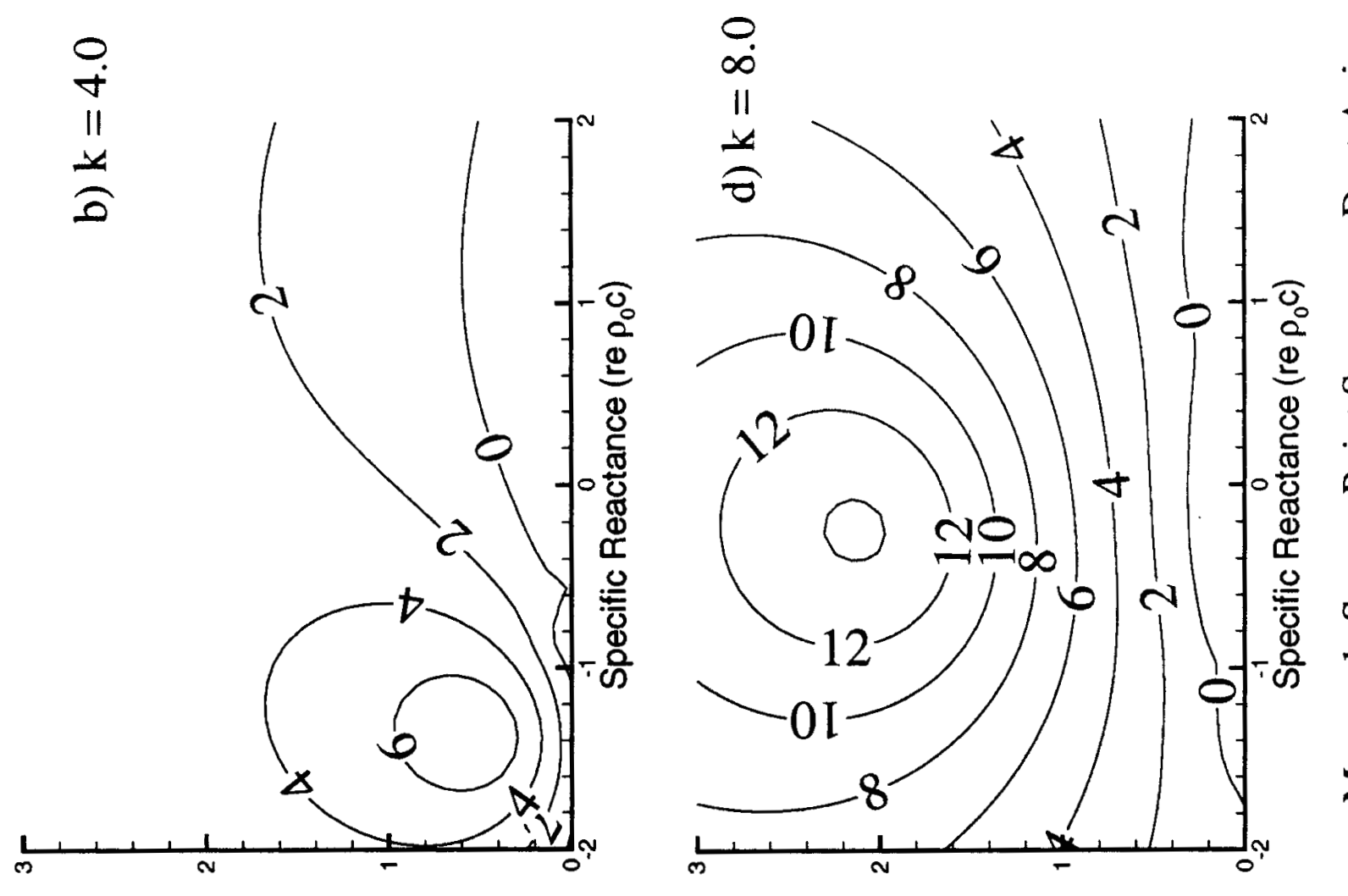

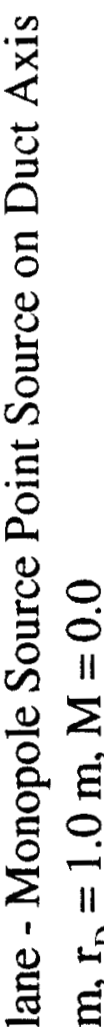

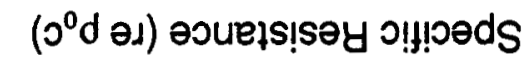

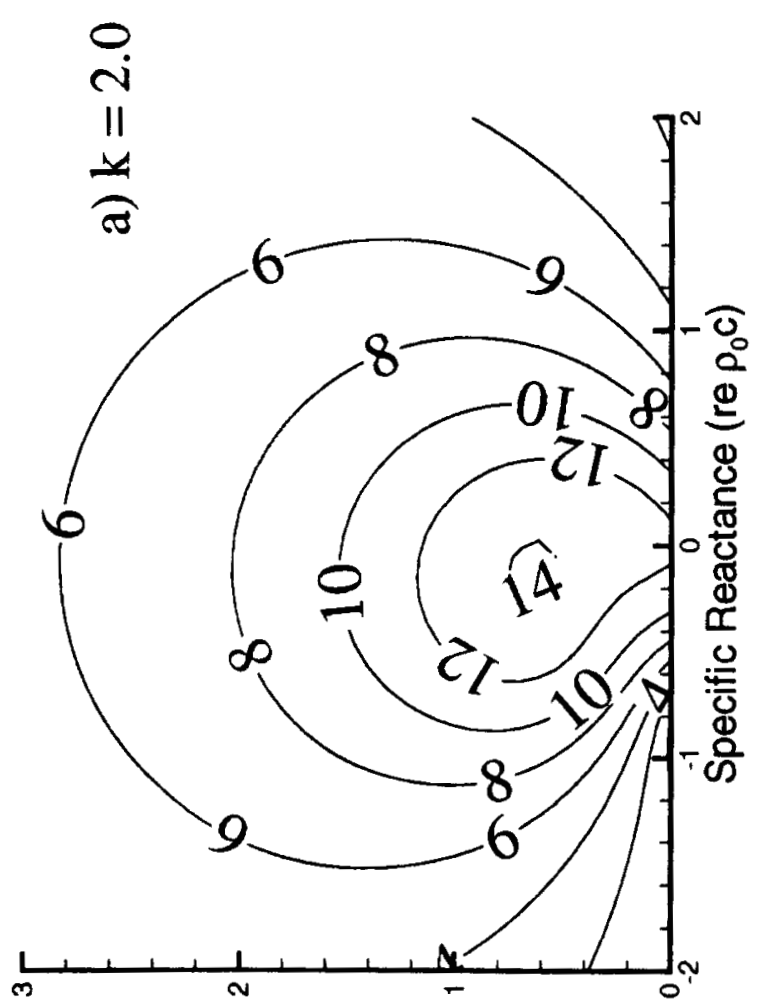

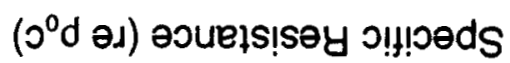

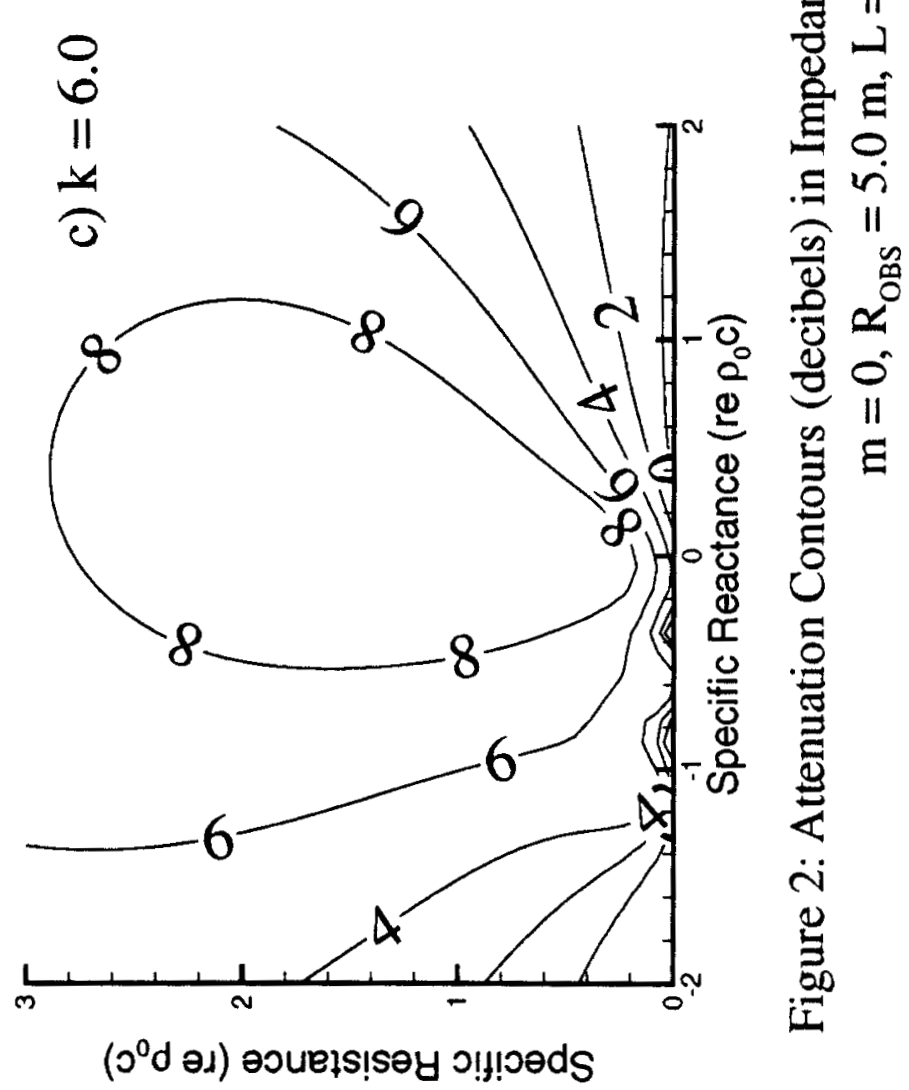



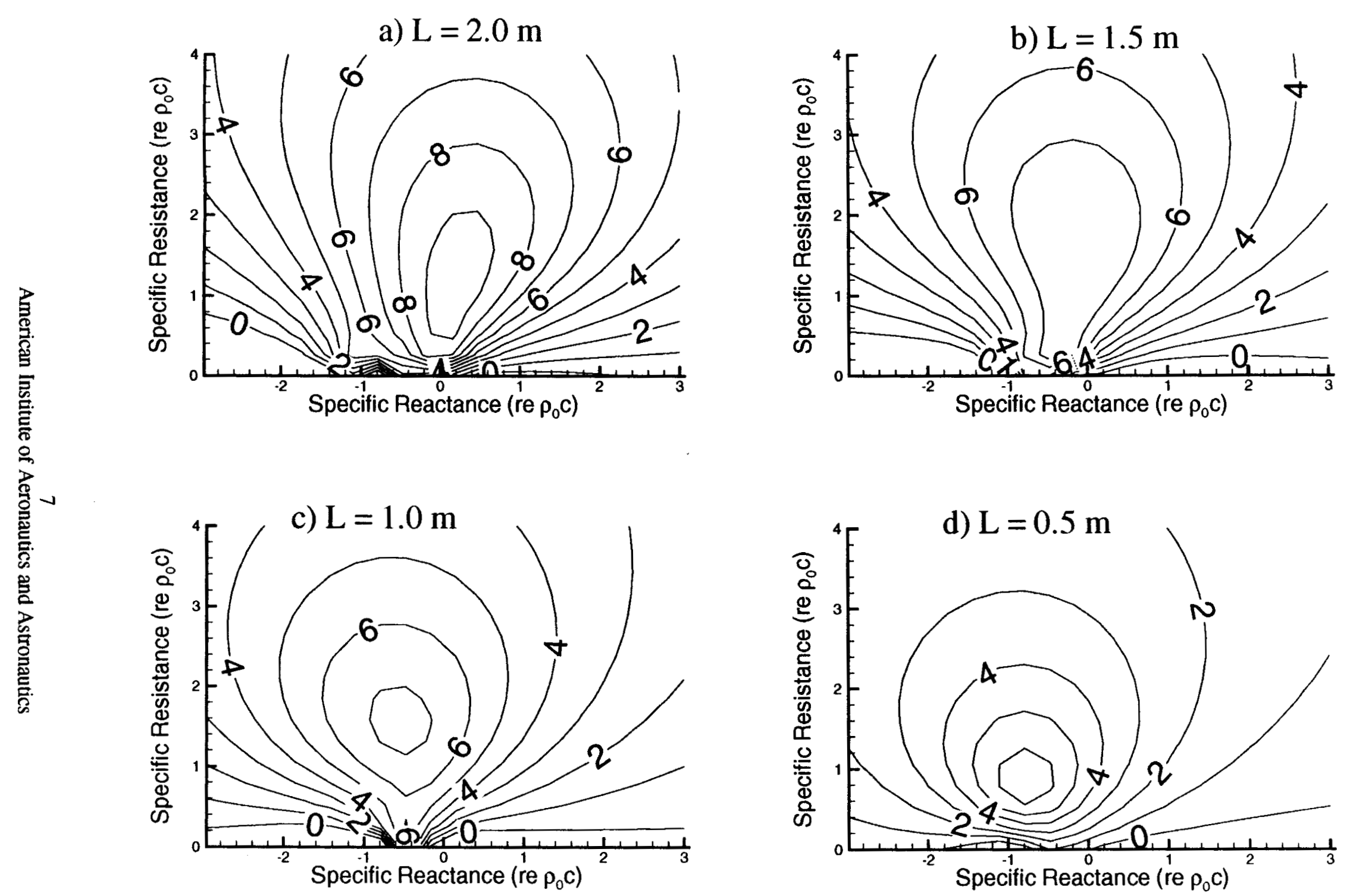

Figure 3: Attenuation as a Function of Liner Length - Monopole Point Source on Duct Axis

$$
\mathrm{m}=0, \mathrm{R}_{\mathrm{OBS}}=5.0 \mathrm{~m}, \mathrm{k}=6, \mathrm{r}_{\mathrm{D}}=1.0 \mathrm{~m}, \mathrm{M}=0.0
$$




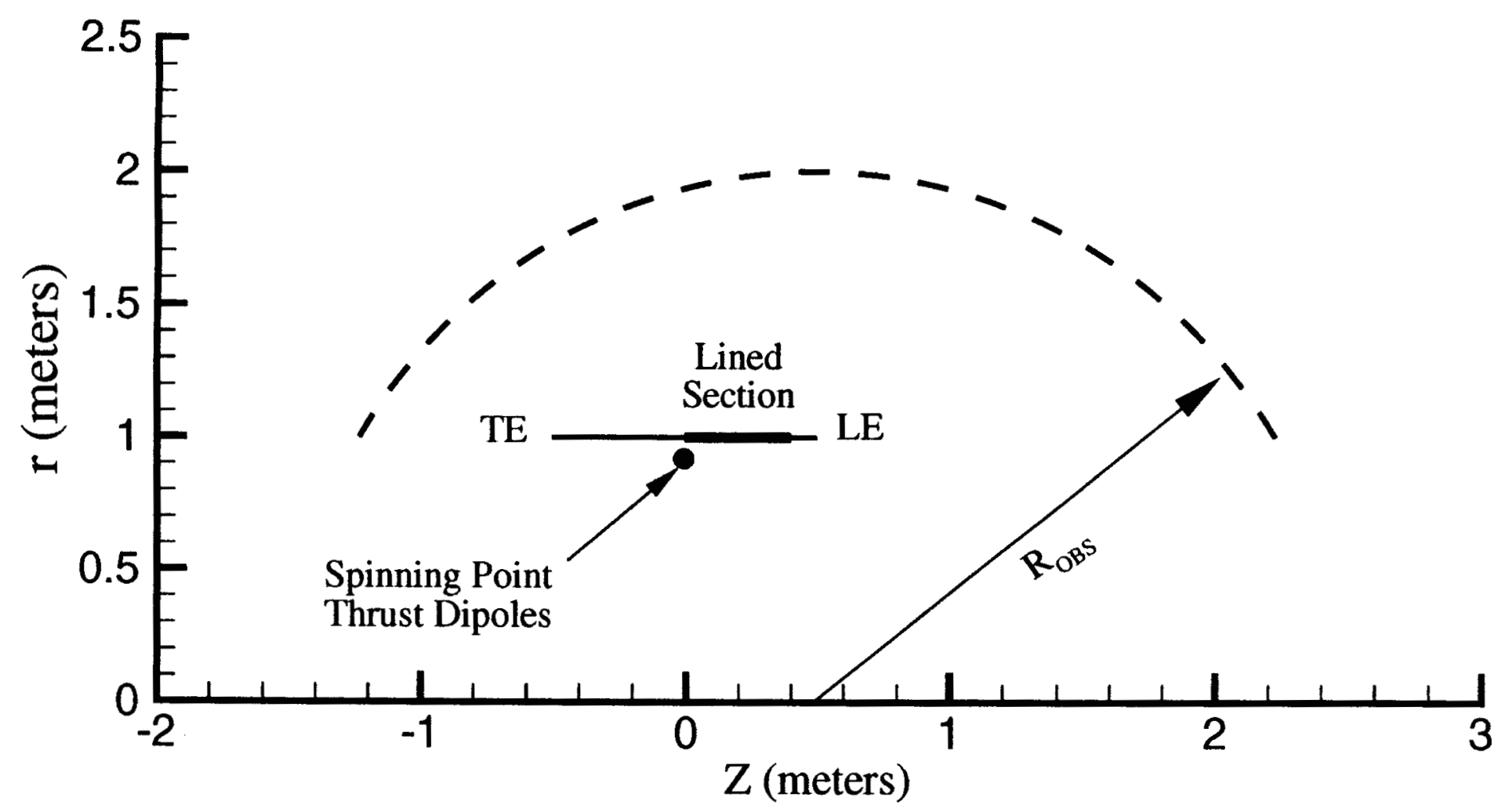

Figure 4: Configuration for Liner Optimization Studies with Spinning Point Thrust Dipole Sources

$$
\begin{array}{clcc}
\mathrm{m}=1 & \mathrm{~N}_{\mathrm{B}}=20 & \mathrm{M}_{\mathrm{TIP}}=1.22 & \mathrm{M}=0.4 \\
\mathrm{~L}_{\mathrm{D}}=0.5 & \mathrm{r}_{\mathrm{D}}=1.0 \mathrm{~m} & \mathrm{~L}=0.4 \mathrm{~m} & \mathrm{R}_{\mathrm{OBS}}=2.0 \mathrm{~m}
\end{array}
$$




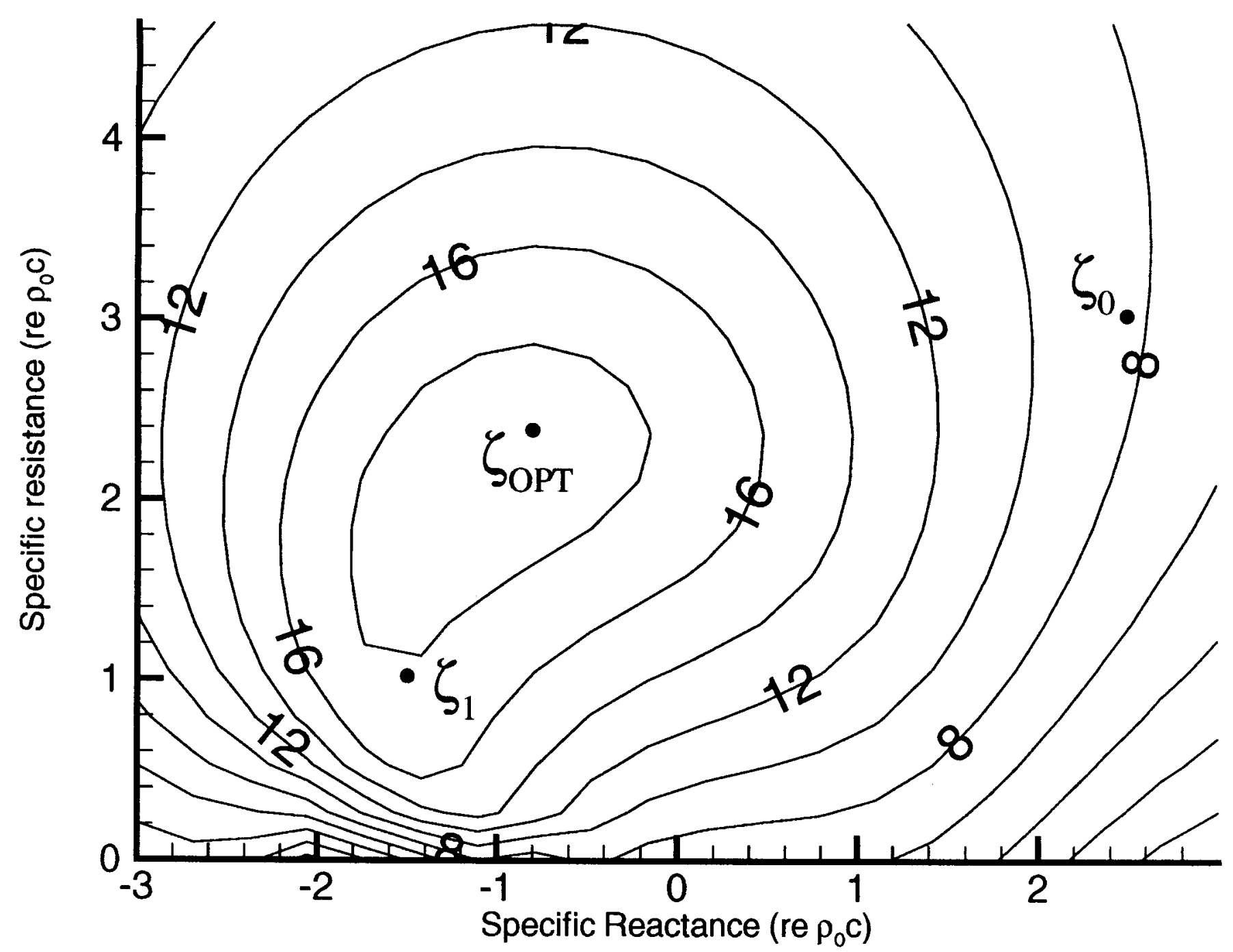

Figure 5: Attenuation Contours in Impedance Plane Spinning Point Thrust Dipole Sources

$$
\begin{gathered}
\mathrm{m}=1, \mathrm{~N}_{\mathrm{B}}=20, \mathrm{M}_{\mathrm{TPP}}=1.22, \mathrm{M}=0.4, \mathrm{~L}_{\mathrm{D}}=0.5, \mathrm{r}_{\mathrm{D}}=1.0 \mathrm{~m}, \mathrm{~L}=0.4 \mathrm{~m} \\
\zeta_{0}=3.00+2.50 \mathrm{i} \quad \zeta_{1}=1.00-1.50 \mathrm{i} \quad \zeta_{\mathrm{OPT}}=2.37-0.79 \mathrm{i}
\end{gathered}
$$



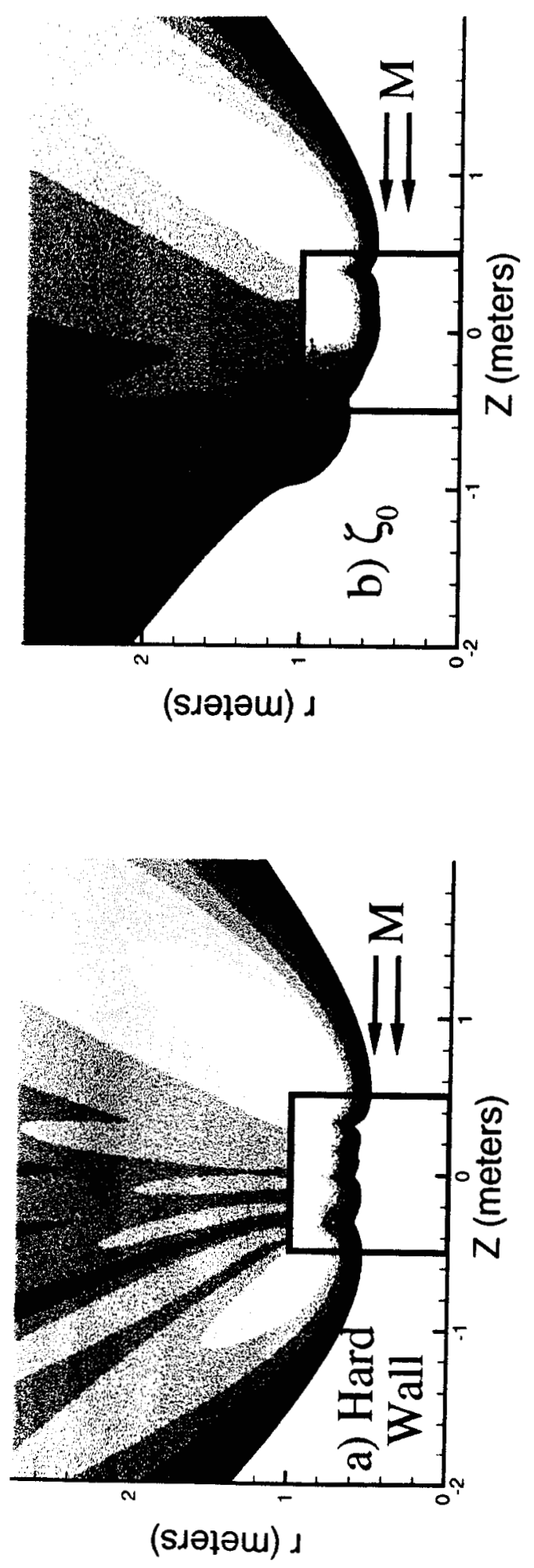

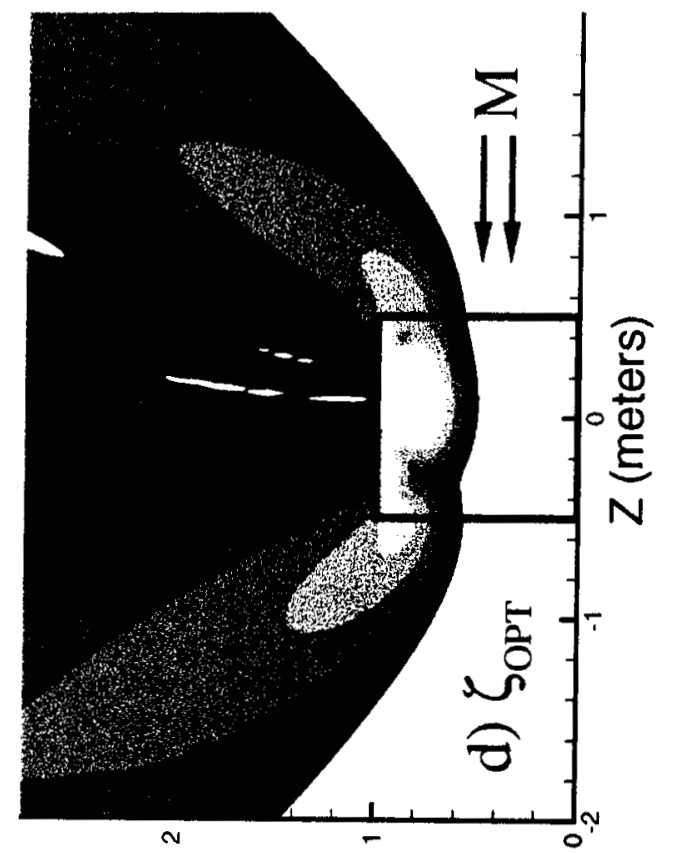

造

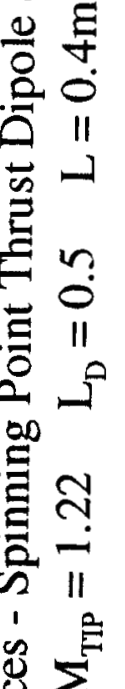

مَ

공

$\Xi$

$\stackrel{\Perp}{N}$

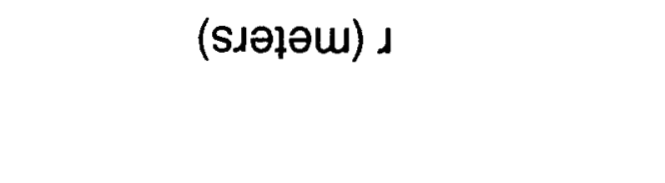

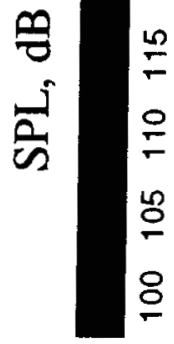

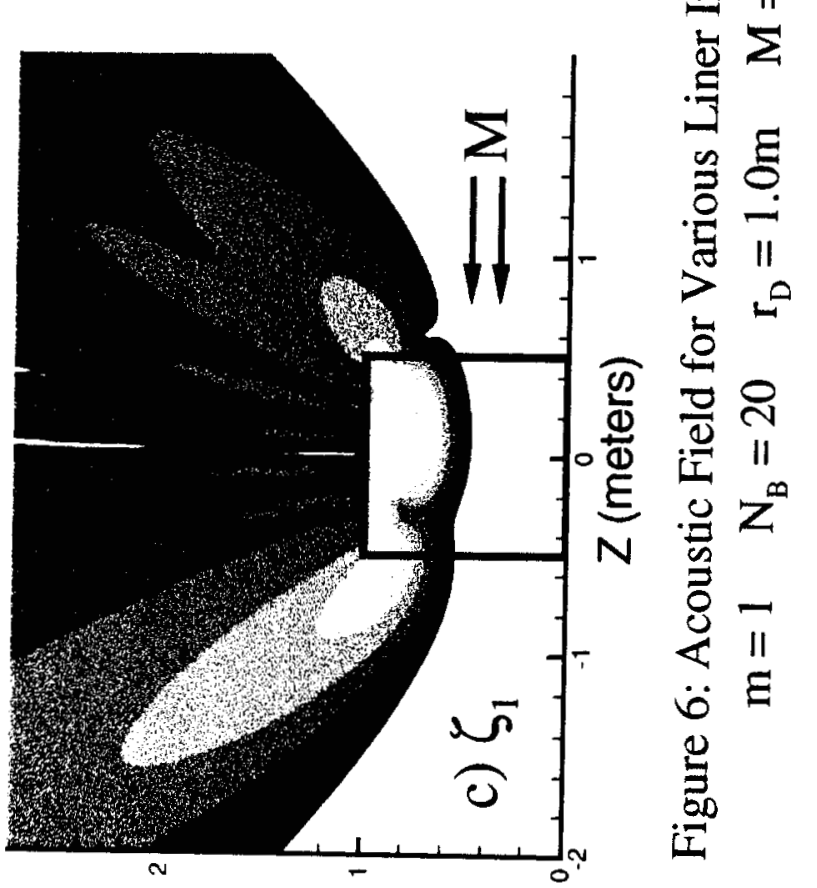

ג 